\title{
Gastric equivalent of the 'Holy Plane' to standardize the surgical concept of stomach cancer to mesogastric excision: updating Jamieson and Dobson's historic schema
}

\author{
Hisashi Shinohara ${ }^{1}\left[\right.$ ' Yasunori Kurahashi ${ }^{1} \cdot$ Yoshinori Ishida $^{1}$
}

Received: 15 September 2020 / Accepted: 14 November 2020 / Published online: 2 January 2021

(c) The International Gastric Cancer Association and The Japanese Gastric Cancer Association 2021

\begin{abstract}
Background Surgery for curable gastric cancer has historically involved dissection of lymph nodes, depending on the risk of metastasis. By establishing the concept of mesogastric excision (MGE), we aim to make this approach compatible with that for colorectal cancer, where the standard is excision of the mesentery.

Methods Current advances in molecular embryology, visceral anatomy, and surgical techniques were integrated to update Jamieson and Dobson's schema, a historical reference for the mesogastrium.

Results The mesogastrium develops with a three-dimensional movement, involving multiple fusions with surrounding structures (retroperitoneum or other mesenteries) and imbedding parenchymal organs (pancreas, liver, and spleen) that grow within the mesentery. Meanwhile, the fusion fascia and the investing fascia interface with adjacent structures of different embryological origin, which we consider to be equivalent to the 'Holy Plane' in rectal surgery emphasized by Heald in the concept of total mesorectal excision. Dissecting these fasciae allows for oncologic MGE, consisting of removing lymph node-containing mesenteric adipose tissue with an intact fascial package. MGE is theoretically compatible with its colorectal counterpart, although complete removal of the mesogastrium is not possible due to the need to spare imbedded vital organs. The celiac axis is treated as the central artery of the mesogastrium, but is peripherally ligated by tributaries flowing into the stomach to feed the spared organs.

Conclusion The obscure contour of the mesogastrium can be clarified by thinking of it as the gastric equivalent of the 'Holy Plane'. MGE could be a standard concept for surgical treatment of stomach cancer.
\end{abstract}

Keywords Mesogastrium $\cdot$ Fusion fascia $\cdot$ Investing fascia $\cdot$ Embryology $\cdot$ Gastric cancer

\section{Introduction}

In 1909, Jamieson and Dobson first reported the arrangement of the lymphatic system of the large intestine [1]. They categorized the lymphatic nodes along the course of the blood vessels into para-colic, intermediate, and main groups. Their schema, as shown in Fig. 1a, is so accurate that it is hard to believe it was developed more than 100 years ago, and it is consistent with the classification system we still use today [2]. They encouraged complete removal of the

Hisashi Shinohara

shinohara@hyo-med.ac.jp

1 Department of Gastroenterological Surgery, Hyogo College of Medicine, 1-1 Mukogawa-cho, Nishinomiya, Hyogo 663-8501, Japan "lymphatic areas" as an excision target in radical surgery for malignant colorectal disease.

In the 1980's, the target changed to encompass the "mesentery". Anchoring the digestive system to the abdominal wall, the mesentery serves as a conduit for blood vessels, lymph vessels, and nerves to reach organs. Initially, it is a simple planar fold protruding from the body wall, but it eventually fuses with somatic structures [3, 4]. Heald proposed the concept of total mesorectal excision (TME) in which the mesorectum was separated, as one lymphovascular entity, by sharp dissection of the areolar interface between the mesentery and its adjacent structures of different embryological origin [5]. He named this space the 'Holy Plane' [6]. Hohenberger later proposed a similar space as a mesenteric plane to achieve complete mesocolic excision (CME) [7]. 
Fig. 1 a Schema of the lymphatics of the colon as described by Jamieson and Dobson in 1909 [1]. b Schema of the lymphatics of the stomach as described by Jamieson and Dobson in 1907 [8]. c Schema of the lymphatic stream of the stomach in the work of Pólya et al. [9]. d Location of lymph node stations as given in Jinnai et al. [11]. Numbers correspond to the lymph node station. e Location of lymph node stations as given in the Japanese Classification of Gastric Carcinoma, 15th edition [13]. f Extent of lymphadenectomy after total gastrectomy as shown in the Japanese Gastric Cancer Treatment Guidelines 2018 (5th edition) [18]
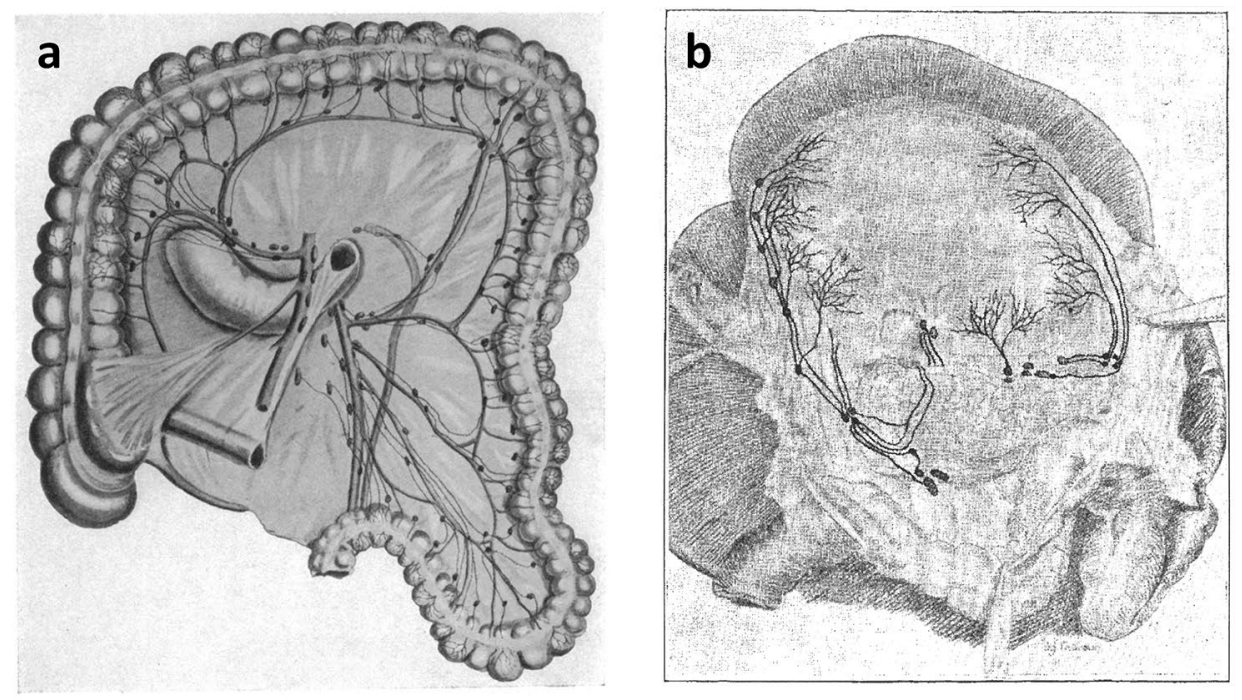

C
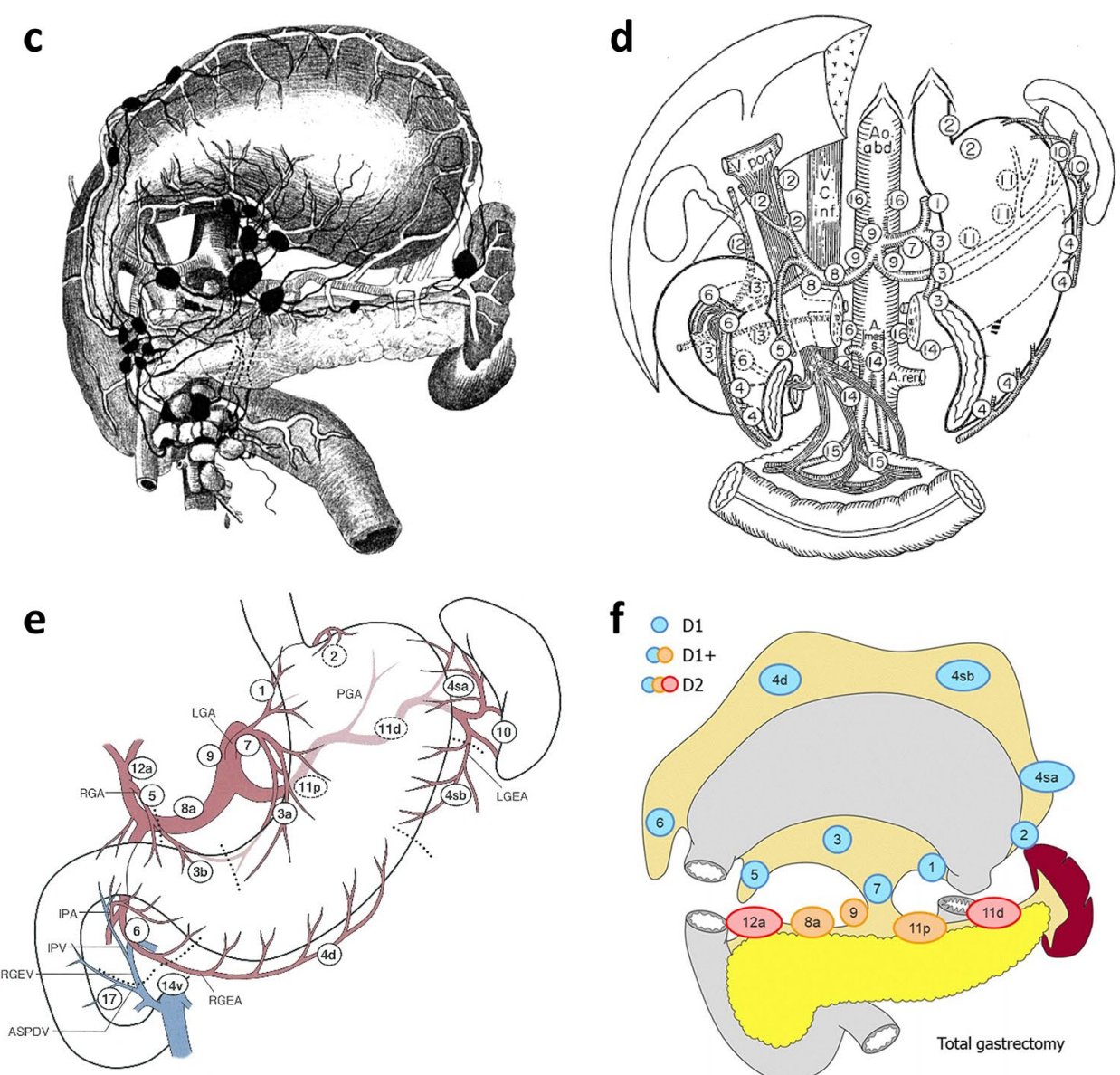

f

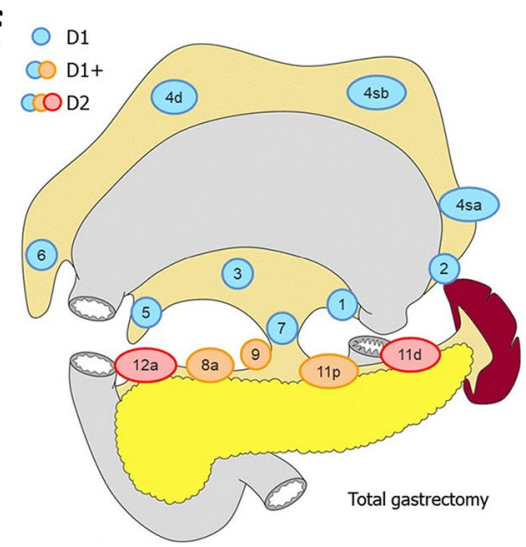

In contrast to this approach to understanding and treating colorectal cancer, the excision target for stomach cancer has not been clearly demarcated. In 1907 in earlier work, Jamieson and Dobson had used the same methodology to trace the lymphatics of the stomach and had published the schema, as shown in Fig. 1b.[8]. They arranged the lymphatic vessels within the mesentery of the stomach-the mesogastrium - which is exposed by lifting the organ after opening the omental bursa. Their paper is clearly a good historical reference, but the schema was not as detailed as that of the large intestine that they published later, probably because it was difficult to distinguish the mesogastrium. Subsequent studies in the early twentieth century led to more detailed descriptions of the lymphatic vessels and nodes 
along with the gastric arteries $[9,10]$, but the mesogastrium was overlooked and no longer outlined, as shown in Fig. 1c. In the 1960's, Jinnai et al. encoded the gastric lymph nodes into 16 stations according to the associated arteries (Fig. 1d) [11]. This recording format has become popular in Japan as a classification system for gastric cancer (Fig. 1e) [12, 13] and is also widely used in Western countries. Subsequently, the frequency of metastasis and the therapeutic efficacy of dissection in each station were scrutinized [14-16], and the extent of lymphadenectomy, such as D1 or D2, was defined by the station code to be dissected, depending on the location and depth of tumor invasion [17-21]. Surgery for curable gastric cancer has therefore long involved dissection of the lymph nodes, classified by risk of metastasis. However, this approach is inconsistent with that for colorectal cancer, where the standard is excision of the mesentery.

Recently, our knowledge of visceral development has grown with the introduction of molecular genetics, as has our knowledge of surgical anatomy under magnified endoscopic and robotic imaging techniques. With these advances, gastric surgeons have begun to realize that the lymph nodes can be resected from landmark structures by meticulously tracing the dissectible layer of loose connective tissue [22-24], a space that could be considered the equivalent of the 'Holy Plane' in rectal surgery. We therefore believe it is now time to universalize this surgical concept across gastrointestinal malignancies. In this article, we bring together current research findings to update Jamieson and Dobson's referential schema of the mesogastrium. After explaining the ambiguity surrounding the mesogastrium, we discuss the rationale for mesogastric excision (MGE) as a standard concept for surgical treatment of stomach cancer, compatible with TME and CME for its colorectal counterpart.

\section{Methods}

\section{Literature review}

The development of the mesogastrium was summarized based on recent findings obtained from image analysis of embryonic and fetal specimens or from molecular genetics that were introduced in the latest editions of representative textbooks of human embryology [3, 4]. Literature on the histology of the mesogastrium were also referenced. Novel findings in visceral anatomy, especially obtained via laparoscopic or robotic gastrectomy and new related techniques, were reviewed and confirmed with our surgical experience.

\section{Artwork}

The collected information and findings were combined to show a series of developmental stages in the mesogastrium including the rotation of the midgut system. Illustrations of sagittal cross-sections to the left of the mesogastrium (along the aorta) and the right of it (along the portal vein) were made to clearly depict avascular interfaces between adjacent structures. The procedure for MGE was then illustrated step by step using the same layout as Jamieson and Dobson's schema [8]. At each step, the interfaces to be identified and dissected are presented in sequence. All figures were drawn by one of the authors (H.S.) using Power Point ${ }^{\mathrm{TM}}$ for Office 365 (Microsoft Corp., Redmond, WA).

\section{Results}

\section{Specific features of the mesogastrium}

The gut tube and its derivatives are suspended from the body wall by mesenteries consisting of double layers of peritoneum that enclose an organ $[3,4]$. The intermediate layer of the mesentery serves as a conduit for blood vessels (arteries and veins), lymphatics (ducts and nodes), and nerves to reach the intestine. After birth, adipose tissue gradually accumulates in this layer, while forming a loose connective tissue space with the peritoneum [25].

In the embryonic stage, the mesogastrium develops with two specific features. The first is a three-dimensional movement involving multiple contacts with surrounding structures. Initially the stomach arising from the foregut has dual planar mesenteries, the dorsal mesogastrium and the ventral mesogastrium (Fig. 2a) [3, 4, 25]. Following gastric rotation, the dorsal mesogastrium inflates toward the left and spatially expands to form the omental bursa and adjoins the parietal wall (Fig. 2b) [26]. The ventral mesogastrium contrarily moves to the right and becomes the lesser omentum. The upcoming anti-clockwise rotation of the midgut loop causes torsion of the dorsal mesogastrium at the gastrocolic trunk (Fig. 2c), followed by contact with the transverse mesocolon (Fig. 2d) [27]. Then, the greater omentum bulges from the omental bursa, and the right extension fuses with the mesoduodenum and right transverse mesocolon (Fig. 2e). The omental bursa expands downward and forms a folded double-layered sac extending over the transverse colon and small intestine as the finale (Fig. 2f) [22, 28, 29]. It is this complicated series of developments that obscures the contours of the mesogastrium.

The second specific feature is the imbedding of several parenchymal organs. The primordium of the pancreas arises from the duodenal wall under regulation of $p d x 1$ and $p t f l a$ [30], grows between the double-layered peritoneum of the mesoduodenum (Fig. 2a), and eventually extends into the dorsal mesogastrium (Fig. 2c) [3]. This event is important because the pancreas is an internal structure within the mesentery of the foregut, but not a retroperitoneal organ like the 
a
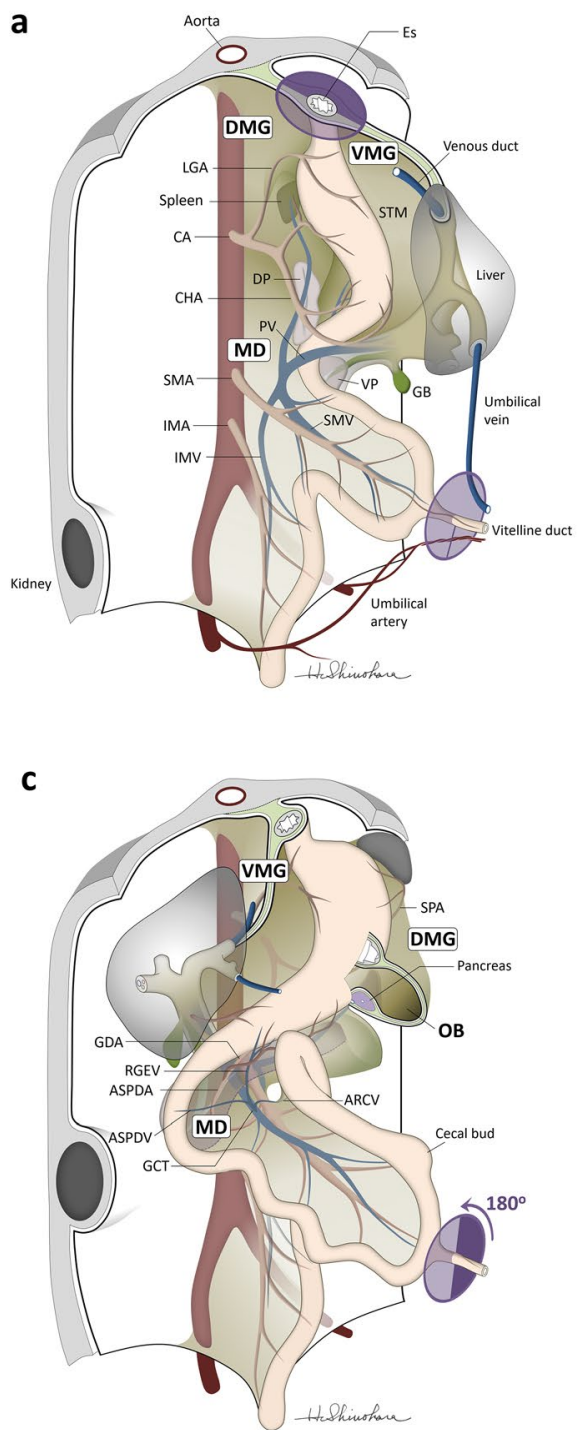

e

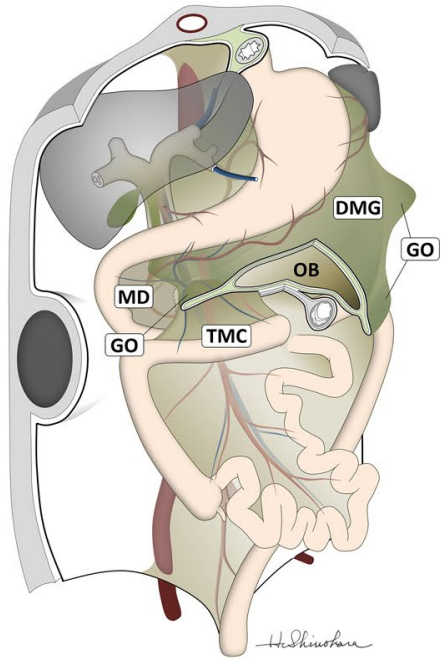

b

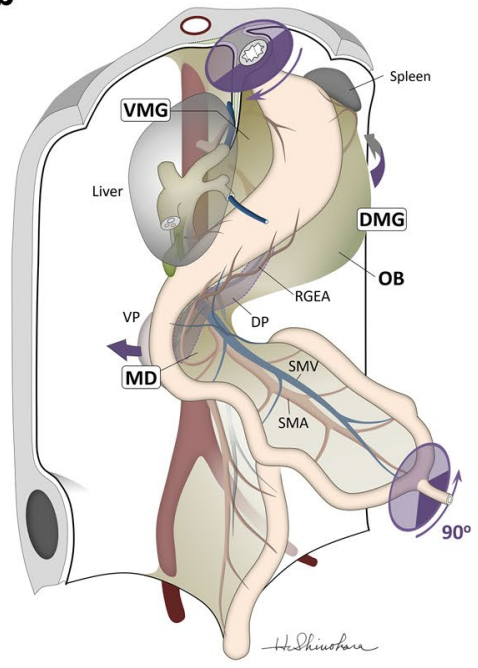

d

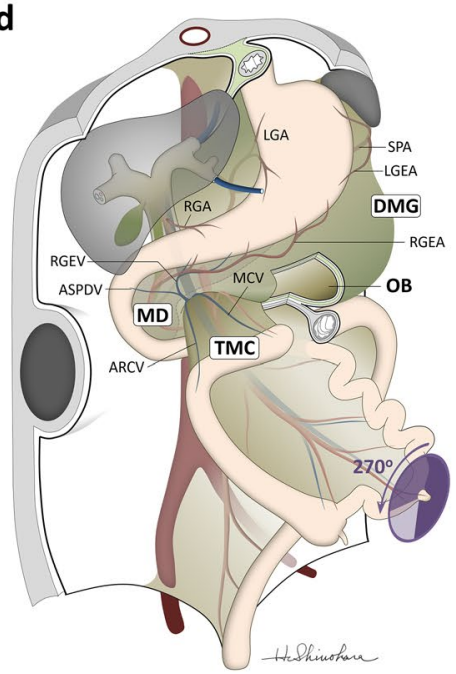

f

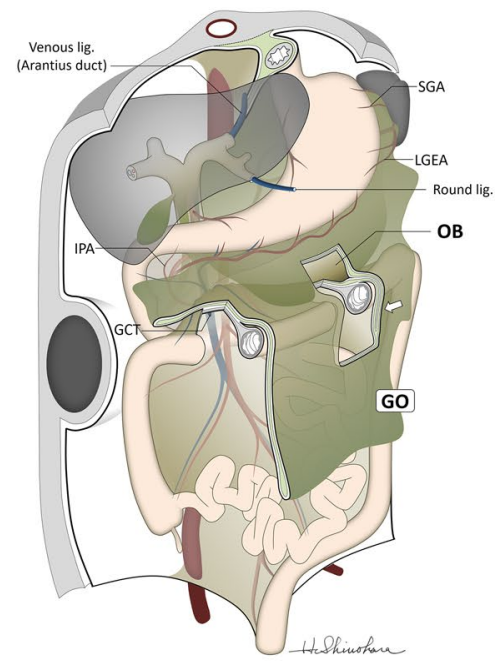


4Fig. 2 Series of developmental stages in the mesogastrium involving rotation of the midgut system. a Primitive gastrointestinal tract and the various derivatives in the fifth week of development. The stomach (STM) is anchored to the abdominal wall via the dorsal mesogastrium (DMG) and the ventral mesogastrium (VMG). The primordium of the pancreas [dorsal pancreas (DP) and ventral pancreas (VP)] arises from the duodenal wall and grows between the layers of the mesoduodenum (MD). The primordium of the liver appears at the distal end of the foregut and grows between the layers of the ventral mesogastrium. The primordium of the spleen appears in the dorsal mesogastrium. b The mesogastrium after 90-degree longitudinal rotation of the stomach and 90-degree counterclockwise rotation of the midgut. The DMG inflates toward the left, expands to form the omental bursa (OB), and adjoins the parietal wall. The VMG contrarily moves to the right and becomes the lesser omentum. c After 180-degree midgut rotation. The pancreas extends into the dorsal mesogastrium. The right gastroepiploic vein (RGEV) and the accessory right colic vein (ARCV) form the gastrocolic trunk (GCT) of Henle and flow into the superior mesenteric vein (SMV). d After completing 270-degree midgut rotation. The DMG contacts with the transverse mesocolon (TMC). The MD is overlaid from the caudal direction with the TMC. e The greater omentum (GO) bulges from the OB. The right extension fuses with the MD and the right TMC. f Fully developed mesogastrium. After expanding downward, the OB forms a folded double-layered sac (arrow) and extends over the transverse colon and small intestine like an apron. ASPDA anterior superior pancreatoduodenal artery, $A S P D V$ anterior superior pancreatoduodenal vein, $C A$ celiac axis, $C H A$ common hepatic artery, Es esophagus, $G B$ gallbladder, GDA gastroduodenal artery, $L G A$ left gastric artery, LGEA left gastroepiploic artery, IMA inferior mesenteric artery, IMV inferior mesenteric vein, $I P A$ infrapyloric artery, $M C V$ middle colic vein, $P V$ portal vein, $R G A$ right gastric artery, $R G E A$ right gastroepiploic artery, $S P A$ splenic artery, $S G A$ short gastric artery

kidney $[3,4,22,28,29]$. The liver primordium appears at the distal end of the foregut and grows in the ventral mesogastrium under regulation of C/EBP alpha [31]. The spleen is also an internal mesenteric structure whose primordium appears in the dorsal mesogastrium under regulation of Hoxl1 [32]. Imbedding these vital organs is a notable limitation placed on the mesogastrium, but not on the mesentery of the large intestine.

\section{Gastric equivalent of the 'Holy Plane'}

During the abovementioned development process, avascular interfaces are formed between the mesogastrium and adjacent structures of different embryological origin. Figure 3 depicts sagittal cross-sections of the mesogastrium along the aorta, showing the omental bursa from inside (Fig. 3a), and along the portal vein, showing the infrapyloric area (Fig. 3b), to demonstrate two groups of interfaces. One is the loose connective tissue space created by contact with the mesothelium of contiguous serosa, and is referred to as the fusion fascia [33]. In CME, the fusion fascia of Toldt (the plane between the ascending mesocolon and the retroperitoneum) [34] and the fusion fascia of Fredet (the plane between the ascending mesocolon and the mesoduodenum) [35] are well-known landmarks. A similar spatial organization is found at the interface between the mesogastrium and adjacent structures such as the retroperitoneum (Fig. 3a, b) [36], the transverse mesocolon (Fig. 3a, b) [27], and the greater omentum (Fig. 3b) [22, 28, 29].

The other is the interface that encloses the imbedding parenchymal organs or vasculature in the mesogastrium. It consists of thin elastic innervated layers of connective tissue, although the thickness varies among the different organs, and is referred to as the investing fascia [33]. It gives the organ shape and supports the parenchyma. As shown in Fig. 3, the investing fascia can be found inside the mesogastrium as a well-defined space interfacing with the pancreas and arteries [22-24, 28, 29], but not with the liver and spleen. These visceral fasciae, the fusion fascia and the investing fascia, provide areolar spaces by which the mesogastric lymph node-containing adipose tissue can be isolated with a connective tissue coating - they are equivalent to the 'Holy Plane' [6].

\section{Greater omentum and omental bursa}

Gastrectomy ordinarily begins with detachment of the greater omentum from the transverse colon. As shown in Fig. 3a, there are several options. Bursectomy comprises removal of the anterior layer of the transverse mesocolon together with the whole greater omentum (thin black arrows) [37], and it may result in maximum resection of the mesogastrium, although its oncological efficacy was refuted by the Japan Clinical Oncology Group 1001 trial [38]. Omentectomy, which is removal of most of the greater omentum by detaching it from the transverse colon (red arrows), is a standard procedure currently. However, the regional lymph nodes of the stomach are known to be located only along the gastroepiploic vessels, not scattered in the peripheral part of the greater omentum [39]. Figure 4a shows a minimal option in which the greater omentum is divided along the gastroepiploic arcade at some distance away to open the omental bursa (white arrow in Fig. 3a). This layout was created to match Jamieson and Dobson's schema. After passing the right edge of the omental bursa, the fusion fascia between the greater omentum and the transverse mesocolon should be dissected (thin black arrows in Fig. 3b).

\section{Infrapyloric area}

The infrapyloric area is in the right border of the mesogastrium adjacent to the mesoduodenum [28]. In the embryonic stage, this area is overlaid from the caudal direction with the transverse mesocolon (Fig. 2d). Thereafter, the greater omentum extending to the right expands over the infrapyloric area and the right transverse mesocolon, as shown in Fig. $2 \mathrm{e}$ and $3 \mathrm{~b}$. To isolate the infrapyloric area, the greater omentum is separated by dissecting the fusion fascia (Fig, 


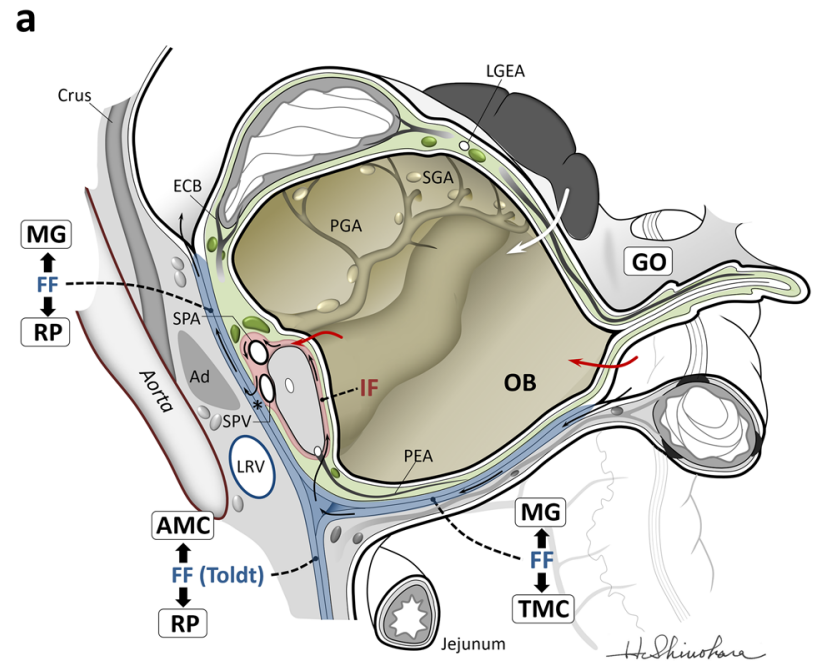

b

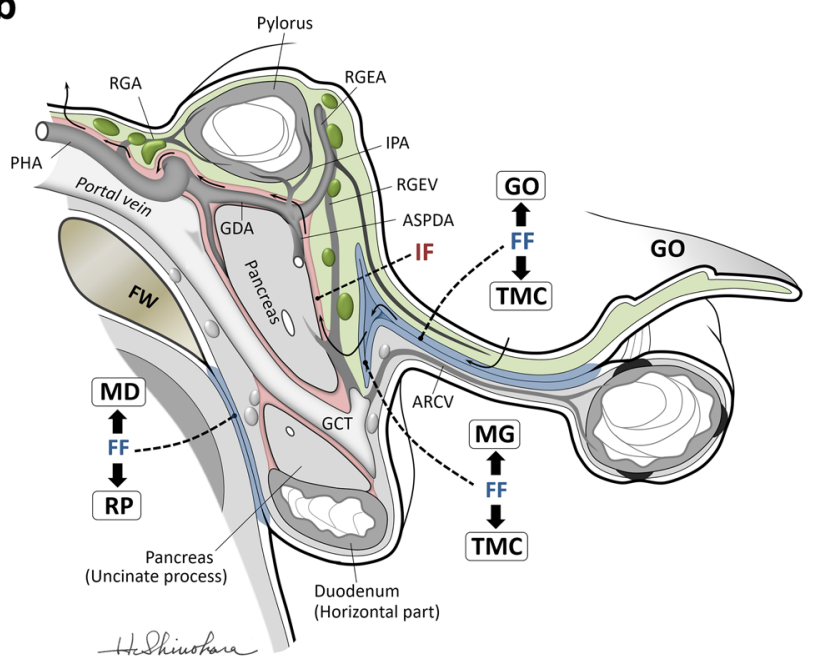

Fig. 3 Sagittal cross-sections of the mesogastrium (MG, green) depicting avascular interfaces consisting of the fusion fascia (FF, blue) and the investing fascia (IF, red) between adjacent structures. a A cross-section along the aorta showing the inflated omental bursa (OB, brown) from inside. Thin black arrows indicate the dissection planes for bursectomy. Note that the IF interfacing with the splenic artery (SPA) continues to the FF $\left(^{*}\right.$ ) between the MG and the retroperitoneum (RP). Thick red arrows indicate the transection lines of the greater omentum (GO) and the suprapancreatic MG to achieve omentectomy. Thick white arrow indicates the transection line to preserve most of the GO. AMC ascending mesocolon, $E C B$ esophago-cardiac branch, $L R V$ left renal vein, $P E A$ posterior epiploic artery, $P G A$ posterior gastric artery, $S G A$ short gastric artery, $S P V$ splenic vein, $T M C$ transverse mesocolon. b Cross-section along the portal vein. The infrapyloric area imbeds the pancreas and is overlaid with the TMC and with the right-extended part of the GO. Thin arrows indicate the dissection planes for infrapyloric lymphadenectomy. Note that the IFs interfacing with the surface of the pancreas, the gastroduodenal artery (GDA), and the proper hepatic artery (PHA) are dissected. $A R C V$ accessory right colic vein, ASPDA anterior superior pancreatoduodenal artery, $F W$ foramen of Winslow, $G C T$ gastrocolic trunk, $I P A$ infrapyloric artery, $M D$ mesoduodenum, $R G A$ right gastric artery, $R G E A$ right gastroepiploic artery, $R G E V$ right gastroepiploic vein
$4 \mathrm{~b})$, then the transverse mesocolon is mobilized by dissecting the fusion fascia (Fig. 4c). Isolating the mesogastrium by prior dissection of the fusion fascia is called "mesenterization" [29].

Due to imbedding of the pancreas, the infrapyloric lymph nodes are located on both its anterior (no. 6) and posterior (no. 13) surfaces [12]. As shown in Figs. 3b and 4c, removal of adipose tissue containing no. 6 lymph nodes is achieved by meticulously dissecting the investing fascia interfacing with the anterior surface of the pancreas [22, 23]. However, to feed the organ, the gastroduodenal artery and the pancreatoduodenal artery are spared by dissecting the investing fascia interfacing with these vessels (see Fig. 3b, thin black arrows), while dividing their branches terminally flowing into the stomach, referred to as the right gastroepiploic artery and the infrapyloric artery, as shown in Fig. 4d [40].

\section{Lesser curvature}

As shown in Fig. 2a, the lesser curvature of the stomach is originally connected to the abdominal wall via the ventral mesogastrium, which later becomes the lesser omentum [3, 4]. The free border of the ventral mesogastrium, the hepatoduodenal ligament, provides a pathway for the hepatobiliary system. The lower-third portion of the lesser curvature is fed by the right gastric artery mostly originated from the proper hepatic artery. Therefore, the lymph nodes of this area categorized as no. $3 \mathrm{~b}$ (along the right gastric artery), no. 5 (root of the right gastric artery), and no. 12 (along the proper hepatic artery) are minor stations belonging to the ventral mesogastrium [41]. Removal of the mesenteric adipose tissue containing the lessor curvature lymph nodes is achieved by dividing the right gastric artery at its root, while the proper hepatic artery is spared to feed the liver by dissecting its investing fascia, as shown in Fig. 3b [23].

\section{Suprapancreatic area}

The celiac artery can be treated as the central artery of the whole mesogastrium. Of its major branches, the common hepatic artery and the splenic artery feed the body of the pancreas. Ligating the celiac artery necessarily entails involvement of the pancreas. However, in a case without direct tumor invasion, combined resection of the pancreas is associated with increased postoperative morbidity and mortality with no survival improvement $[42,43]$. Thus, the pancreas is a vital organ to be spared when performing standard gastrectomy, even though it is imbedded in the mesogastrium. As shown in Fig. 4e, removal of the mesenteric adipose tissue containing the suprapancreatic lymph nodes (nos. $7,8,9,11$ ) is achieved by dividing the left gastric artery, the posterior gastric artery, and the left gastroepiploic artery at their root, while sparing the celiac artery and its 
major branches, the common hepatic artery, and the splenic artery to feed the pancreas by dissecting the investing fascia interfacing with these vessels (see Fig. 3a, thin black arrows). [23] As indicated by the asterisk in Fig. 3a, the investing fascia interfacing with the splenic artery continues to the fusion fascia lying between the mesogastrium and the retroperitoneum, which was formed after inflation of the dorsal mesogastrium toward the left parietal wall. Prior dissection of this fusion fascia allows isolation of the mesogastrium (mesenterization) [29], resulting in deliberate lymph node dissection along the splenic artery [44].

\section{Splenectomy and splenic hilar node dissection}

Even without direct tumor invasion to the parenchyma, splenectomy has been performed as a standard procedure for D2 total gastrectomy that is aimed at complete nodal dissection at the splenic hilum (no. 10) [17]. This situation contrasts with that in combined pancreatectomy, because the disadvantages posed by splenectomy were thought to be negligible. However, a recent randomized controlled trial demonstrated that splenectomy increases operative morbidity without improving survival for proximal gastric cancer that does not invade the greater curvature [45], and the no. 10 station was subsequently removed from the definition of D2 lymph node dissection in total gastrectomy [18]. It is theoretically possible to remove only the adipose tissue containing no. 10 lymph nodes if dissecting the investing fascia interfacing with the peripheral branches of the splenic artery, but its oncological benefit is not known.

\section{Removing the mesogastrium as an intact entity}

Figure 4f shows the final view of the steps in MGE. Dissected lymph nodes are involuntarily included within one of the compartments of the mesogastrium. The dissection plane of the excised specimen, which had been interfacing with the surrounding structures, is covered with connective tissue of the separated visceral fasciae, the fusion fascia and the investing fascia. This is the same for the dissection plane on the remaining side; the spared viscera including the pancreas and major arterial branches of the celiac axis are seen through the fascial coating.

\section{Discussion}

The mesogastrium is a complex mesentery that develops with a three-dimensional movement, involving multiple fusions and imbedding of vital organs. This is likely why the concept of mesenteric resection has not been accepted in gastric cancer surgery. Recently, in magnified images obtained by laparoscopic or robotic gastrectomy, surgeons have begun to acknowledge there is a dissectible loose connective-tissue layer between the lymph nodes and landmark structures. As described in this article, this is actually equivalent to the 'Holy Plane' [6]: identifying and dissecting these planes allows oncologic MGE, which consists of removing the mesenteric adipose tissue containing the lymph nodes as an intact fascial package. Thus, gastric lymphadenectomy can be achieved by MGE, which is theoretically compatible with the concepts of TME and CME [5, 7]. However, MGE must be performed systematically, but not completely, due to the need to spare imbedded organs and major branches of the celiac axis [29].

D2 gastrectomy has become the best recommended curative treatment for gastric cancer [46]. To standardize the procedure, the extent of lymphadenectomy was defined by encoded regional lymph nodes depending on the risk of metastasis [11-15]. In the third edition of the Japanese guidelines for treatment of gastric cancer published in 2010, the lymph nodes to be dissected were defined according to the type of gastrectomy and not the location of the tumor or depth of invasion [17]. Also, as shown in Fig. 1f, the number of lymph node stations was indicated as a compartment of fat attachment surrounding the stomach, rather than overlying the accompanying arteries. Notably, the contour of the attachment matches the shape of the specimen from MGE shown in Fig. 4f, suggesting that the resection target of gastrectomy encompasses from the lymph nodes to the mesentery. In that sense, the concept of MGE is applicable not only to standard D2 gastrectomy but also to D1 or D1+ gastrectomy, because the extent of lymphadenectomy can be modified by compartments of the mesogastrium [47].

We acknowledge that MGE is not a novel surgical procedure but rather a concept that provides a theoretical background to established gastric cancer surgery. In other words, MGE is not an extension, but rather the ideal form, of D2 gastrectomy performed under accurate identification and meticulous dissection of the visceral fasciae. Therefore, MGE will not allow for increased number of retrieved lymph nodes, nor will it dramatically improve prognostic outcomes compared with well-established D2 surgery. However, available evidence suggests that removing the lymph nodecontaining adipose tissue while leaving the fascial package intact may reduce postoperative complications [22, 28, 47]. Etoh et al. reported that extranodal metastasis, defined as the presence of cancer cells in adipose tissue, was detected in nearly $40 \%$ of patients with T4a tumors who underwent radical gastrectomy [48]. Cancer cells may well spill into the peritoneal cavity from breached fascia during lymphadenectomy [49]. As already demonstrated in TME [50], locoregional control could be improved under the concept of MGE.

The concept of MGE we advocate here is based on anatomical understanding acquired in open surgery and was also refined through knowledge acquired during laparoscopic and 
a

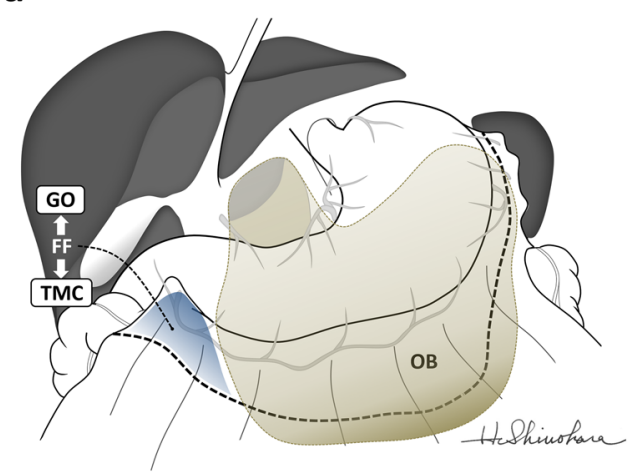

c

e

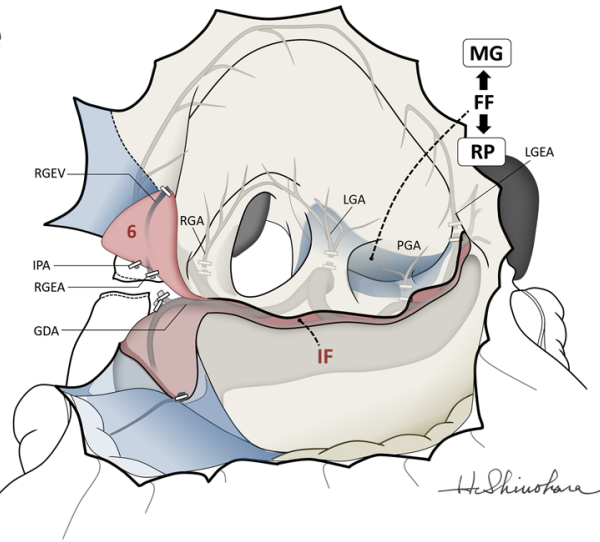

b

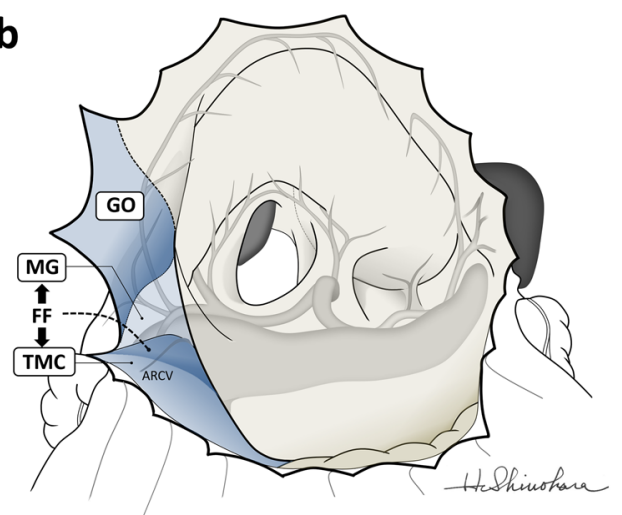

d

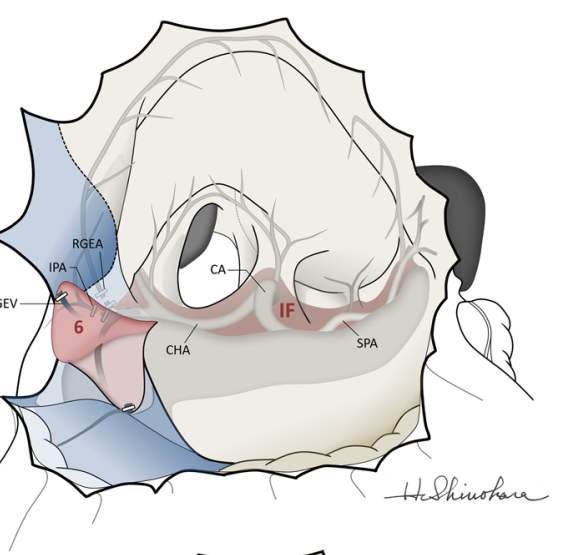

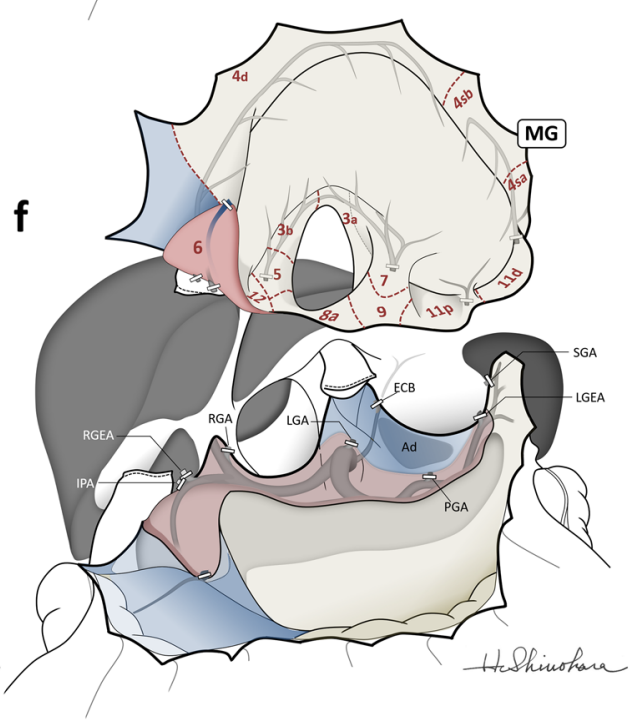

robotic surgery. We believe the concept confirms the rationality of D2 gastrectomy that experienced elderly surgeons perform and thus can be passed on to younger surgeons with confidence as well as also be useful for minimally invasive surgery that they are interested in. However, standard surgery comprises only part of the multidisciplinary treatment required for gastric cancer in its various stages. We anticipate that surgical oncology will be further advanced by integration with extended gastrectomy for highly advanced gastric cancer that has progressed beyond the boundaries of the mesentery or with chemotherapy to downstage the tumor to achieve curability.

\section{Conclusion}

The often obscure contour of the mesogastrium can be clarified by thinking of it as the gastric equivalent of the 'Holy Plane'. MGE could be a standard concept for 
४Fig. 4 Procedure of mesogastric excision (MGE) outlined step by step. At each step, the interfaces, consisting of the fusion fascia (FF, blue) and the investing fascia (IF, red), are presented in sequence. The layout matches that of Jamieson and Dobson's schema (Fig. 1b). a Detachment of the greater omentum (GO) from the transverse colon by the right gastroepiploic vessels at some distance away (dashed line) to open the omental bursa (OB, brown). The transparent plane of the FF between the GO and the transverse mesocolon (TMC) is subsequently dissected. b After separating the GO. The transparent plane of the FF between the TMC and the infrapyloric area of the mesogastrium (MG) is subsequently dissected. ARCV, accessory right colic vein. c After mobilizing the TMC. The transparent plane of the IF interfacing with the pancreas is subsequently dissected. ASPDA, anterior superior pancreatoduodenal artery; RGEV, right gastroepiploic vein. d After removing the MG containing the no. 6 infrapyloric lymph nodes. The RGEV, the right gastroepiploic artery (RGEA), and the infrapyloric artery (IPA) are divided. The transparent plane of the IF interfacing with the pancreas, the celiac axis (CA) and its major branches feeding the pancreas, and the liver is subsequently dissected. CHA common hepatic artery, PHA proper hepatic artery, SPA the splenic artery. e After removing the MG containing the suprapancreatic lymph nodes. The right gastric artery (RGA), the left gastric artery (LGA), the posterior gastric artery (PGA), and the left gastroepiploic artery (LGEA) are divided. The transparent plane of the FF between the MG and the retroperitoneum (RP) continued from the suprapancreatic IF is indicated. GDA gastroduodenal artery. f After completing MGE. Note the dissection planes on both the excised MG and the remaining side are covered with connective tissue of the separated visceral fasciae. Numbers in the MG represent the lymph node stations. Ad adrenal gland, ECB esophago-cardiac branch, SGA short gastric artery

surgical treatment of stomach cancer, theoretically compatible with the colorectal counterpart for TME and CME. We propose that surgical resection for gastric cancer with curative intent be performed under the concept of MGE.

Acknowledgements The authors thank Takeshi Sano for informative discussions and Caryn Jones for professional editing and language revision.

Funding This work was supported in part by Japan Society for the Promotion of Science KAKENHI Grant Number 19H03735. The sponsor had no role in study design, data collection, data analysis, manuscript preparation, or publication decisions.

\section{Compliance with ethical standards}

Conflict of interest The authors declare no conflict of interest.

Ethical approval All procedures performed in studies involving human participants were in accordance with the ethical standards of the institutional and/or national research committee and with the 1964 Helsinki declaration and its later amendments or comparable ethical standards.

Informed consent Informed consent was obtained from all individual participants included in the study.

\section{References}

1. Jamieson JK, Dobson JF. The lymphatics of the colon: with special reference to the operative treatment of cancer of the colon. Ann Surg. 1909;50:1077-90.

2. Japanese Society for Cancer of the Colon and Rectum. Japanese classification of colorectal, appendiceal, and anal carcinoma: the 3rd English edition. J Anus Rectum Colon. 2019;3:175-95.

3. Sadler TW, Langman J. Langman's medical embryology. 14th ed. Philadelphia: Lippincott Williams \& Wilkins; 2019.

4. Moore KL, Persaud TVN, Torchia MG. The developing human: clinically oriented embryology. 8th ed. Philadelphia: Saunders/ Elsevier; 2008.

5. Heald RJ, Husband EM, Ryall RDH. The mesorectum in rectal cancer surgery: The clue to pelvic recurrence? Br J Surg. 1982;69:613-6.

6. Heald RJ. The 'Holy Plane' of rectal surgery. J Royal Soc Med. 1988;81:503-8.

7. Hohenberger W, Weber K, Matzel K, Papadopoulos T, Merkel S. Standardized surgery for colonic cancer: complete mesocolic excision and central ligation-technical notes and outcome. Colorectal Dis. 2009;11:354-64.

8. Jamieson JK, Dobson JF. Lectures on the lymphatic system of the stomach. Lancet. 1907;169:1061-6.

9. Pólya E, von Navratil D. Untersuchung úber die Lymphbahnen des Wurmfortsatzes und des Magens. Zeitschr für klin Chir. 1903;69:421-56.

10. Rouvière H. Anatomie des lymphatiques de l'homme. Paris: Masson; 1932. p. 294-334.

11. Jinnai D, Kajitani T, Kuru M, Maki T, Murakami T, Mutoh M, et al. Explanation about "Classification of Gastric Carcinoma in surgery.” Syujutsu. 1963;17:951-9 ((Japanese)).

12. Japanese Gastric Cancer Association. Japanese classification of gastric carcinoma: 3rd English edition. Gastric Cancer. 2011a;14:101-12.

13. Japanese Gastric Cancer Association. Japanese classification of gastric carcinoma. 15th ed. Tokyo: Kanehara; 2017.

14. Maruyama K, Gunvén P, Okabayashi K, Sasako M, Kinoshita T. Lymph node metastases of gastric cancer. General pattern in1931 patients. Ann Surg. 1989;210:596-602.

15. Sasako M, McCulloch P, Kinoshita T, Maruyama K. New method to evaluate the therapeutic value of lymph node dissection for gastric cancer. Br J Surg. 1995;82:346-51.

16. Hanna GB, Amygdalos I, Ni M, Boshier PR, Mikhail S, Lloyd J, et al. Improving the standard of lymph node retrieval after gastric cancer surgery. Histopathology. 2013;63:316-24.

17. Japanese Gastric Cancer Association. Japanese gastric cancer treatment guidelines 2010 (ver. 3). Gastric Cancer. $2011 b ; 14: 113-23$.

18. Japanese Gastric Cancer Association. Japanese gastric cancer treatment guidelines 2018 (5th edition). Gastric Cancer. 2020. https://doi.org/10.1007/s10120-020-01042-y.

19. De Manzoni G, Marrelli D, Baiocchi GL. The Italian Research Group for Gastric Cancer (GIRCG) guidelines for gastric cancer staging and treatment: 2015. Gastric Cancer. 2017;20:20-30.

20. NCCN Clinical Practice Guidelines in Oncology. Gastric Cancer Version 3; 2015. http://www.nccn.org/professionals/physi cian_gls/pdf/gastric.pdf. Accessed 31 Aug 2020.

21. Waddell T, Verheij M, Allum W, Cunningham D, Cervantes A, Arnold D. Gastric cancer: ESMO-ESSO-ESTRO clinical practice guidelines for diagnosis, treatment and follow-up. Ann Oncol. 2013;24(Suppl 6):vi57-63.

22. Shinohara H, Haruta S, Ohkura Y, Udagawa H, Sakai Y. Tracing dissectable layers of mesenteries overcomes embryologic restrictions when performing infrapyloric lymphadenectomy 
in laparoscopic gastric cancer surgery. J Am Coll Surg. 2015;220:e81-7.

23. Shibazaki S, Suda K, Nakauchi M, Nakamura T, Kadoya S, Kikuchi K, et al. Outermost layer-oriented medial approach for infrapyloric nodal dissection in laparoscopic distal gastrectomy. Surg Endosc. 2018;32:2137-48.

24. Noshiro H, Ikeda O, Urata M. Robotically-enhanced surgical anatomy enables surgeons to perform distal gastrectomy for gastric cancer using electric cautery devices alone. Surg Endosc. 2014;28:1180-7.

25. Shinohara H. Anatomy of the stomach and surrounding structures. In: Illustrated abdominal surgery: based on embryology and anatomy of the digestive system. Springer: Singapore; 2020. pp. $1-19$

26. Nakamura T, Yamada S, Funatomi T, Takakuwa T, Shinohara H, Sakai Y. Three-dimensional morphogenesis of the omental bursa from four recesses in staged human embryos. J Anat. 2020. https ://doi.org/10.1111/joa.13174.

27. Jeong YJ, Cho BH, Kinugasa Y, Song CH, Hirai I, Kimura W, et al. Fetal topohistology of the mesocolon transversum with special reference to fusion with other mesenteries and fasciae. Clin Anat. 2009;22:716-29.

28. Shinohara H, Kurahashi Y, Kanaya S, Haruta S, Ueno M, Udagawa $\mathrm{H}$, et al. Topographic anatomy and laparoscopic technique for dissection of no6 infrapyloric lymph nodes in gastric cancer surgery. Gastric Cancer. 2013;16:615-20.

29. Shinohara H, Kurahashi Y, Haruta S, Ishida Y, Sasako M. Universalization of the operative strategy by systematic mesogastric excision for stomach cancer with that for total mesorectal excision and complete mesocolic excision colorectal counterparts. Ann Gastroenterol Surg. 2018;2:28-36.

30. Kawaguchi Y, Cooper B, Gannon M, Ray M, MacDonald RJ, Wright CV. The role of the transcriptional regulator Ptf1a in converting intestinal to pancreatic progenitors. Nat Genet. 2002;32:128-34

31. Shiojiri N, Takeshita K, Yamasaki H, Iwata T. Suppression of C/ EBP alpha expression in biliary cell differentiation from hepatoblasts during mouse liver development. J Hepatol. 2004;41:790-8.

32. Roberts CWM, Shutter JR, Korsmeyer SJ. Hox 11 controls the genesis of the spleen. Nature. 1994;368:747-9.

33. Stecco C, Sfriso MM, Porzionato A, Rambaldo A, Albertin G, Macchi V, et al. Microscopic anatomy of the visceral fasciae. J Anat. 2017;231:121-8.

34. Culligan K, Sehgal R, Mulligan D, Dunne C, Walsh S, Quondamatteo $\mathrm{F}$, et al. A detailed appraisal of mesocolic lymphangiology —an immunohistochemical and stereological analysis. J Anat. 2014;225:463-72.

35. Garcia-Granero A, Pellino G, Frasson M, Fletcher-Sanfeliu D, Bonilla F, Sánchez-Guillén L, et al. The fusion fascia of Fredet: an important embryological landmark for complete mesocolic excision and D3-lymphadenectomy in right colon cancer. Surg Endosc. 2019;33:3842-50.

36. Kitagawa H, Tajima H, Nakagawara H, Hayashi H, Makino I, Takamura $\mathrm{H}$, et al. The retropancreatic fusion fascia acts as a barrier against infiltration by pancreatic carcinoma. Mol Clin Oncol. 2013;1:418-22.

37. Groves EW. On the radical operation for cancer of the pylorus: with especial reference to the advantages of the two-stage operation and to the question of the removal of the associated lymphatics. Br Med J. 1910;1:366-70.
38. Kurokawa Y, Doki Y, Mizusawa J, Terashima M, Katai H, Yoshikawa $\mathrm{T}$, et al. Bursectomy versus omentectomy alone for resectable gastric cancer (JCOG1001): a phase 3, open label, randomized controlled trial. Lancet Gastroenterol Hepatol. 2018;3:460-8.

39. Liebermann-Meffert $\mathrm{D}$, White $\mathrm{H}$. The greater omentum. Berlin: Springer-Verlag; 1983.

40. Haruta H, Shinohara H, Ueno M, Udagawa H, Sakai Y, Uyama I. Anatomical considerations of the infrapyloric artery and its associated lymph nodes during laparoscopic gastric cancer surgery. Gastric Cancer. 2015;18:876-80.

41. Haruta S, Shinohara H, Hosogi H, Ohkura Y, Kobayashi N, Mizuno A, et al. Proximal gastrectomy with exclusion of no $3 \mathrm{~b}$ lesser curvature lymph node dissection could be indicated for patients with advanced upper-third gastric cancer. Gastric Cancer. 2017;20:528-35.

42. Degiuli M, Sasako M, Calgaro M, Garino M, Rebecchi F, Mineccia M, et al. Morbidity and mortality after D1 and D2 gastrectomy for cancer: interim analysis of the Italian Gastric Cancer Study Group (IGCSG) randomised surgical trial. Eur J Surg Oncol. 2004;30:303-8.

43. Bonenkamp JJ, Songun I, Hermans J, Sasako M, Welvaart K, Plukker JT, et al. Randomised comparison of morbidity after D1 and D2 dissection for gastric cancer in 996 Dutch patients. Lancet. 1995;345:745-8.

44. Kumamoto T, Kurahashi Y, Niwa H, Nakanishi Y, Ozawa R, Okumura $\mathrm{K}$, et al. Laparoscopic suprapancreatic lymph node dissection using a systematic mesogastric excision concept for gastric cancer. Ann Surg Oncol. 2020;27:529-31.

45. Sano T, Sasako M, Mizusawa J, Yamamoto S, Katai H, Yoshikawa $\mathrm{T}$, et al. Randomized controlled trial to evaluate splenectomy in total gastrectomy for proximal gastric carcinoma. Ann Surg. 2017;265:277-83

46. Songun I, Putter H, Kranenbarg EM, Sasako M, van de Velde CJ. Surgical treatment of gastric cancer: 15 -year follow-up results of the randomized nationwide Dutch D1 D2 trial. Lancet Oncol. 2010;19:439-49.

47. Kumamoto T, Kurahashi Y, Haruta S, Niwa H, Nakanishi Y, Ozawa R, et al. Laparoscopic modified lymphadenectomy in gastric cancer surgery using systematic mesogastric excision: a novel technique based on a concept. Langenbecks Arch Surg. 2019;404:369-74.

48. Etoh T, Sasako M, Ishikawa K, Katai H, Sano T, Shimoda T. Extranodal metastasis is an indicator of poor prognosis in patients with gastric carcinoma. Br J Surg. 2006;93:369-73.

49. Xie D, Wang Y, Shen J, Hu J, Yin P, Gong J. Detection of carcinoembryonic antigen in peritoneal fluid of patients undergoing laparoscopic distal gastrectomy with complete mesogastric excision. Br J Surg. 2018;105:1471-9.

50. Heald RJ, Santiago I, Pares O, Carvalho C, Figueiredo N. The perfect total mesorectal excision obviates the need for anything else in the management of most rectal cancers. Clin Colon Rectal Surg. 2017;30:324-32.

Publisher's Note Springer Nature remains neutral with regard to jurisdictional claims in published maps and institutional affiliations. 